\title{
Multi-Granular Optical Cross-Connect: Design, Analysis, and Demonstration
}

\author{
Georgios S. Zervas, Marc De Leenheer, Lida Sadeghioon, Dimitris Klonidis, Yixuan Qin, \\ Reza Nejabati, Dimitra Simeonidou, Chris Develder, Bart Dhoedt, and Piet Demeester
}

\begin{abstract}
A fundamental issue in all-optical switching is to offer efficient and cost-effective transport services for a wide range of bandwidth granularities. This paper presents multi-granular optical crossconnect (MG-OXC) architectures that combine slow (ms regime) and fast (ns regime) switch elements, in order to support optical circuit switching (OCS), optical burst switching (OBS), and even optical packet switching (OPS). The MG-OXC architectures are designed to provide a cost-effective approach, while offering the flexibility and reconfigurability to deal with dynamic requirements of different applications. All proposed MG-OXC designs are analyzed and compared in terms of dimensionality, flexibility/ reconfigurability, and scalability. Furthermore, node level simulations are conducted to evaluate the performance of MG-OXCs under different traffic regimes. Finally, the feasibility of the proposed architectures is demonstrated on an application-aware, multi-bitrate (10 and $40 \mathrm{Gbps}$ ), end-to-end OBS testbed.
\end{abstract}

Index Terms-Multi-granular optical cross-connect; Optical circuit switching; Optical burst switching; Simulation analysis; Demonstration.

\section{INTRODUCTION}

A generation of distributed network-based applications that combine scientific instruments, distributed data archives, sensors, computing resources, and many others are emerging. Each application has its own traffic profile, resource usage pattern, and different requirements originating in the computing, stor-

Manuscript received January 9, 2009; accepted January 12, 2009; published June 1, 2009 (Doc. ID 110468).

G. S. Zervas, L. Sadeghioon, R. Nejabati, Y. Qin, and D. Simeonidou are with the Photonic Networks Research Lab, School of Computer Science and Electronic Engineering, University of Essex, Wivenhoe Park, CO4 3SQ Colchester, UK (e-mail: gzerva@essex.ac.uk).

M. De Leenheer, C. Develder, B. Dhoedt, and P. Demeester are with the Department of Information Technology, Ghent University-IBBT, Gaston Crommenlaan 8 bus 201, 9050 Gent, Belgium (e-mail: marc.deleenheer@intec.ugent.be).

D. Klonidis is with the Athens Information Technology Centre, Athens, Greece.

Digital Object Identifier 10.1364/JOCN.1.000069 age, and network domains [1]. Dedicated networks do not offer sufficient flexibility to satisfy the requirements of each application type, nor are they economically acceptable. Hence it is vital to understand and redefine the role of networking, to support applications with different requirements, and also offer service providers a flexible, scalable, and cost-effective solution. A dynamic optical network infrastructure with the ability to provide bandwidth granularity at different levels is a potential candidate. In this way, the network can adapt to application requirements and also support different levels of quality of service (QoS). However, care must be taken to devise a solution that remains scalable and cost-effective (see also Section II.C).

A multi-granular optical switched network is able to support dynamic wavelength and sub-wavelength bandwidth granularities with different QoS levels. As such, the network will support the three basic switching technologies in WDM networks: optical circuit switching (OCS), optical packet switching (OPS), and optical burst switching (OBS).

In OCS networks, bandwidth granularity is at the wavelength level since one or more wavelengths are allocated to a connection, while connectivity between the source and destination is established using a twoway reservation, which is in general a timeconsuming procedure. The OCS scheme is suitable for applications that need continuous bandwidth for a relatively long duration of time. However, this scheme is neither sufficiently flexible nor bandwidth efficient to support applications that require sub-wavelength bandwidth granularity in an on-demand fashion or for a short duration of time.

OPS technology makes it possible to achieve subwavelength bandwidth granularity and exploit statistical multiplexing of bursty traffic flows. In OPS networks, one or more IP packets with similar attributes are aggregated in an optical packet and tagged with an optical header. This scheme does not need advance reservation, and it can provide on-demand connectivity by imitating best-effort IP packet routing in the optical domain. These features make OPS technology a suitable candidate for applications that need trans- 
mission of small data sets in an on-demand manner [2]. However, the lack of practical optical buffering solutions, together with the high cost of switch fabrics that meet the constraints imposed by OPS, make this technology difficult to implement and deploy in practice.

OBS combines the advantages of OCS and OPS [3]. The fundamental premise is the separation of the control and data planes, and the segregation of functionality within the appropriate domain (electronic or optical). Prior to data transmission, a burst control header $(\mathrm{BCH})$ is created and sent towards the destination. The $\mathrm{BCH}$ is typically sent out-of-band over a separate signaling wavelength and processed electronically at intermediate OBS routers. It informs each node of the impending arrival of the data burst, and in turn drives the allocation of an optical path. Data bursts remain in the optical plane end-to-end and are typically not buffered as they transit the network core. The main advantages of OBS are that, unlike OCS networks, the optical bandwidth is reserved only for the duration of the actual data transfer, and thus OBS increases bandwidth efficiency. Likewise, in contrast to OPS networks, OBS is not based on besteffort routing and potentially can be implemented without buffers.

In order to migrate from a strictly wavelength granular network (OCS) to a sub-wavelength one (OBS or even OPS) and/or also consider the possibility of hybrid OCS/OBS type of networks (Fig. 1), switching technologies with speeds on a millisecond range down to the nanosecond range must be considered. The network resource reservation can be realized by first sending a reservation message (e.g., RSVP Path message for GMPLS or LOBS [4], BCH for OBS) to the network. The control mechanism then recognizes whether the data unit requires slow switching (usually long burst or circuit) or fast switching (usually short burst or packet). In the first case, the slow switch element is dynamically reconfigured so that when the long data set arrives, it is routed to the appropriate output port. In the other case, the short data unit (burst or packet) is routed through the fast switch, either by directly connecting incoming wavelengths or using pre-established soft-permanent connections through the slow switch to allow reconfigurability (see Section III for more details).

OCS can utilize millisecond switching technologies efficiently, whereas this switching speed causes bandwidth inefficiency and unpredictability for the performance of OBS, especially under high network load. This is mainly caused by the high overhead incurred by large offset times required to configure slow switches. Indeed, the throughput of a switch is defined as $/ T_{\text {offset }}+T_{\text {data }}$, where $T_{\text {data }}$ is the length of the actual data in time, and $T_{\text {offset }}$ is the offset time between the control message and the data. MEMS switches (microelectro-mechanical systems) typically have a switching time of around $10 \mathrm{~ms}$, and thus provide a throughput of more than $95 \%$ on a 10 Gbps system with data duration of $200 \mathrm{~ms}$. If only $1 / 10$ th of this data is sent (which still amounts to 25 MBytes of data), then the

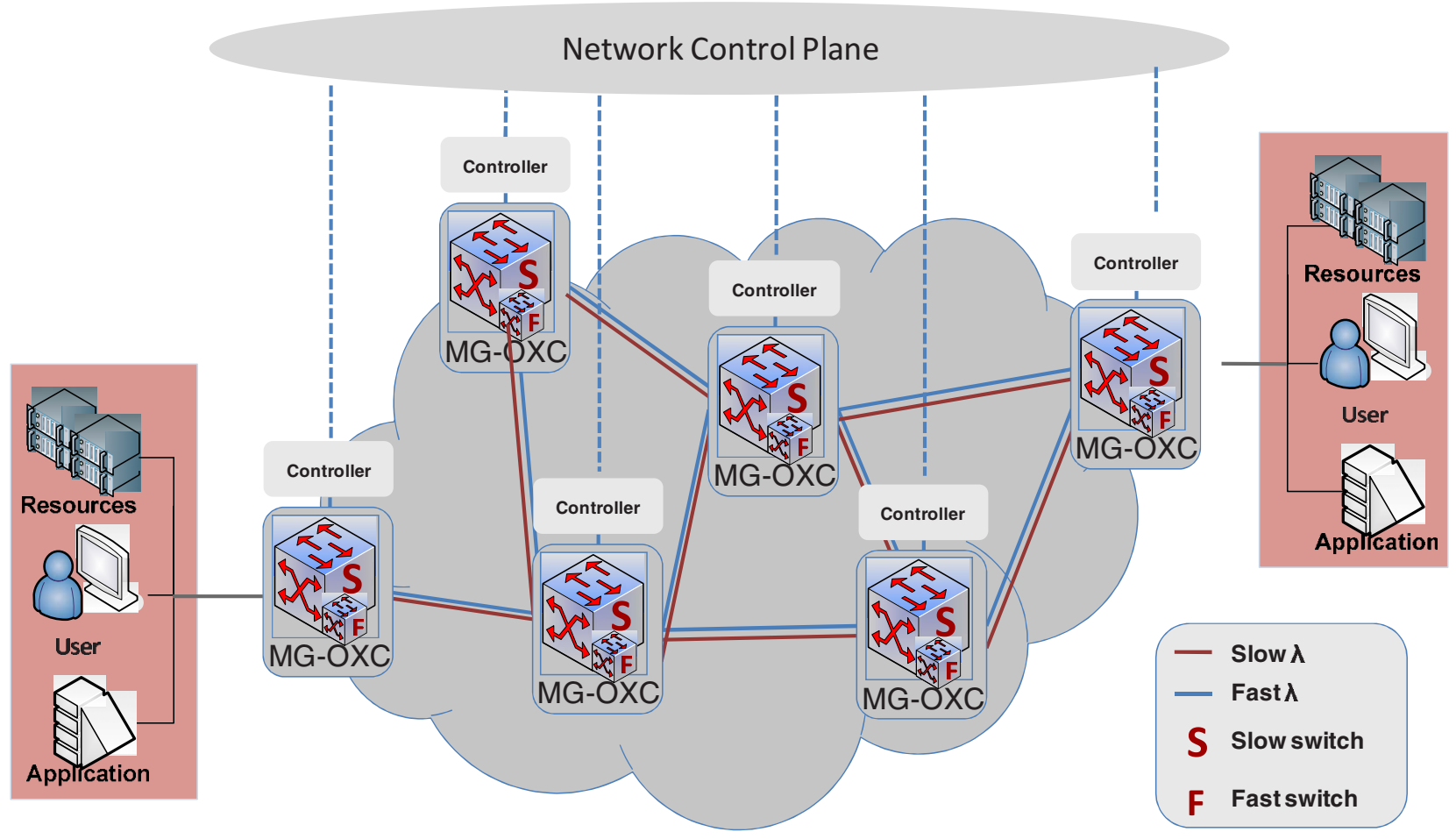

Fig. 1. (Color online) Multi-granular optical network environment. 
switch's throughput decreases to $67 \%$. This effect becomes even more severe when the bandwidth is increased, for instance, to 40 or even $160 \mathrm{Gbps}$, or when OPS (data durations on the nanosecond scale) must be supported. An SOA-based switch (semiconductor optical amplifier) can achieve nanosecond switching speeds and is thus much better adapted to support the full range of data sizes required for OCS, OBS, and OPS.

The ideal solution would thus consist of deploying fast switches of large dimension and low cost; however, these do not exist at this time (a brief review of existing switching technologies is provided in Section II.A). Therefore, one possible solution is an optical cross-connect (OXC) architecture that combines both slow (e.g., MEMS) and fast (e.g., SOA-based) switching elements. This way, users and applications can decide on slow or fast network provisioning, and additionally the network service provider can optimize bandwidth utilization by allocating wavelengths or lightpaths according to the traffic's switching needs. Furthermore, our previous work [22] has shown the cost-effectiveness of the proposed approach on the network level.

In conclusion, the multigranular OXC (MG-OXC) has a number of distinct advantages over traditional single-fabric switches:

- Bandwidth provisioning and switching capability at fiber, wavelength, and sub-wavelength granularities;

- Agility and scalability of switching granularities providing a dynamic solution;

- Fast reconfigurability and flexibility on the electronic control of switching technologies;

- Cost-performance efficiency by offering an optimal balance between slow and fast switch fabric technologies.

The remainder of this paper is organized as follows. We first review existing switch fabric technologies for possible deployment (Section II.A) and then discuss alternative proposals for multi-granular optical switching in Section II.B. Section III proposes different MG-OXC architectures and presents an analysis on the dimensionality, scalability, and flexibility of the designs. Simulations are used to evaluate the MGOXC for various traffic and design parameters in Section IV. Furthermore, in Section V, we demonstrate an MG-OXC based on MEMS for circuits or long bursts and SOA-MZI for medium to short bursts or optical packets. Results are shown for an end-to-end, application-aware, multi-bit-rate and QoS-enabled (based on bandwidth and latency parameters) OBS network testbed. Finally, Section VI summarizes our conclusions.

\section{RELATED WORK}

\section{A. Optical Switching Technologies}

This section briefly reviews the most prominent switching technologies available and comments on their usability in different network environments (OCS, OBS, OPS).

Three main categories considered for optical switch fabric selection [5] are a) signal quality, b) configuration, and c) physical performance. Each category has a number of attributes [6], which are listed below:

- Signal quality is largely determined by the bit error rate (BER) and the cascadability of the switch fabric. Important parameters are signal-to-noise ratio, jitter, amplitude distortion, crosstalk, and extinction ratio.

- Configuration is mostly related to the implementation of the switch and is determined by its scalability, blocking characteristics, and costeffectiveness. Further design considerations include control requirements, dynamic range, polarization dependence, degree of multicast, latching/non-latching, promptness (store-andforward versus cut-through), upgradeability, power requirements, and size.

- Finally, the physical performance of a switch is determined by optical bandwidth, switching speed, insertion loss, bit rate limit, bit pattern dependence, uniformity, and optical transparency.

In [7], Baldine et al. realized an OBS testbed using MEMS OXCs from ATDnet. In [8,9], NTT and Fujitsu demonstrated burst switching with GMPLS-based two-way signaling protocol, utilizing planar lightwave circuit (PLC) and MEMS switches. In [10], commercial PLC switches were adopted to construct a 1616 non-blocking switch matrix with a switching speed of less than $3 \mathrm{~ms}$. OBS schemes with hybrid optical and electrical switching technologies have been investigated in the past [11]. A recent study demonstrated that a PLZT switch (lead lanthanum zirconate titanate, [12]) with shared wavelength conversion allowed variable-length $3.5 \mathrm{~ns}$ OBS. A photonic random access memory (RAM) was used in [13] to write and read high-speed asynchronous burst optical packets. In this paper, the main criteria used in the decision process are the switching time and scalability. Thus, according to Yoo et al. in [5], Table I identifies possible switch fabrics for the development of a multi-granular switch. A detailed discussion based on the attributes mentioned above can be found in Wei et al. [11] and Papadimitriou et al. [6]. Clearly, one of the most appropriate slow switching technologies is the optical MEMS switch, since it is already a mature technology under production and because of the high dimensions available at low cost $[14,15]$. On the other hand, most fast switch matrices (ns regime) listed in Table I are 
TABLE I

Switch Fabrics for OCS, OBS, ANd OPS BASEd on Switching Time AND Scalability

\begin{tabular}{lccc}
\hline & Switching time & Scalability & Applications \\
\hline Opto-mechanical switch & $4 \mathrm{~ms}$ & $16 \times 16$ & OCS, limited OBS \\
Optical MEMS & $3 \mathrm{D}: \sim 10 \mathrm{~ms}, 2 \mathrm{D}: \sim 3 \mathrm{~ms}$ & $3 \mathrm{D}: 1000 \times 1000,2 \mathrm{D}: 32 \times 32$ & OCS, limited OBS \\
Thermo-optical PLC & $\sim 3 \mathrm{~ms}$ & $4 \times 4$ & OCS, limited OBS \\
PLZT switch & $\sim 20 \mathrm{~ns}$ & $4 \times 4$ & OCS, OBS, OPS \\
Semiconductor optical phase array & $\sim 30 \mathrm{~ns}$ & $64 \times 64$ & OCS, OBS, OPS \\
Wavelength routing switch & $\sim 1 \mathrm{~ns}$ & $65,336 \times 65,336$ & OCS, OBS, OPS \\
SOA broadcast-and-select & $\sim 1 \mathrm{~ns}$ & $32 \times 32$ & OCS, OBS, OPS \\
SOA cross-point & $\sim 1 \mathrm{~ns}$ & $4 \times 4$ & OCS, OBS, OPS \\
Optical RAM & $\sim 1 \mathrm{~ns}$ & $64 \times 64$ & OCS, OBS, OPS \\
\hline
\end{tabular}

still in the research stage; some are not yet integrated into a single device (e.g., wavelength routing switch) or are highly sensitive to polarization (e.g., SOA-based switches). As such, it is not yet possible to select the most appropriate technology at this stage.

\section{B. Multi-Granular Optical Cross Connects}

The principle of multi-granular optical crossconnects that can switch traffic at fiber, waveband, and wavelength granularities [16-18] has been proposed to reduce the cost and complexity of traditional OXCs. The MG-OXC is a key element for routing high speed WDM data traffic in a multi-granular optical network. A three-layer switching fabric consisting of a fiber cross-connect (FXC), a band cross-connect (BXC), and a wavelength cross-connect (WXC) was presented in $[19,20]$, and the application of such three-layer MGOXC architectures to metro-area networks was demonstrated in [21]. The work presented in this paper can be considered as complementary to the studies mentioned above. Instead of extending the granularity towards higher capacities (e.g., wavebands to full fibers), we propose OXC architectures that are able to switch at the sub-wavelength level. By combining the two extensions, the full range of bandwidth granularities can be supported by a single OXC design.

\section{Cost Effectiveness}

In our previous work [22], we have shown that multi-granular switching also provides economic advantages on the network level, which provides further motivation for the cost-effectiveness of the proposed approach. For this, an ILP-based network dimensioning algorithm was introduced to compare multigranular switching with approaches based on a single technology (MEMS for slow switching, SOA for fast). Results indicated that significant cost savings can be obtained when implementing multi-granular optical switching. Another important conclusion was that the reconfigurable MG-OXC design (see Section III.A) leads to only a negligable cost increase compared to the non-reconfigurable design. Furthermore, reduced node costs can be achieved as well, in order to minimize scalability problems corresponding to emerging fast switching fabrics.

\section{Multi-Granular Optical CROSS CONNECT ARCHITECTURES}

This section presents, analyzes and evaluates different architectures aiming to support OCS, OBS, and OPS (or even a combination of them, e.g., hybrid OCS/ OBS) with a single design.

\section{A. MG-OXC Design}

Before discussing the proposed switch designs, we state our design goals. We first note that neither wavelength conversion nor buffering capabilities are available, as these technologies are both costly and impractical.

- Each design must support multi-granular optical switching and consist of both slow and fast switching fabrics.

- The architectures must be non-blocking in the sense that any input wavelength can be connected to any output fiber. The stricter form where any input wavelength can be connected to any output wavelength is not achievable since we assume no wavelength conversion is available.

- A number of wavelengths of each fiber should be able to access the fast switch, although only limited fiber-wavelength pairs can do so simultaneously.

- We favor designs that allow the set of fiberwavelength pairs, which have access to the fast switch, to be reconfigurable. However, we also show switch designs in which this functionality can only be achieved through manual intervention. In the following paragraph, we explain reconfigurability in more detail.

- The design should be able to scale in fast or slow switch ports depending on network requirements (i.e., adding new wavelengths and/or links) 


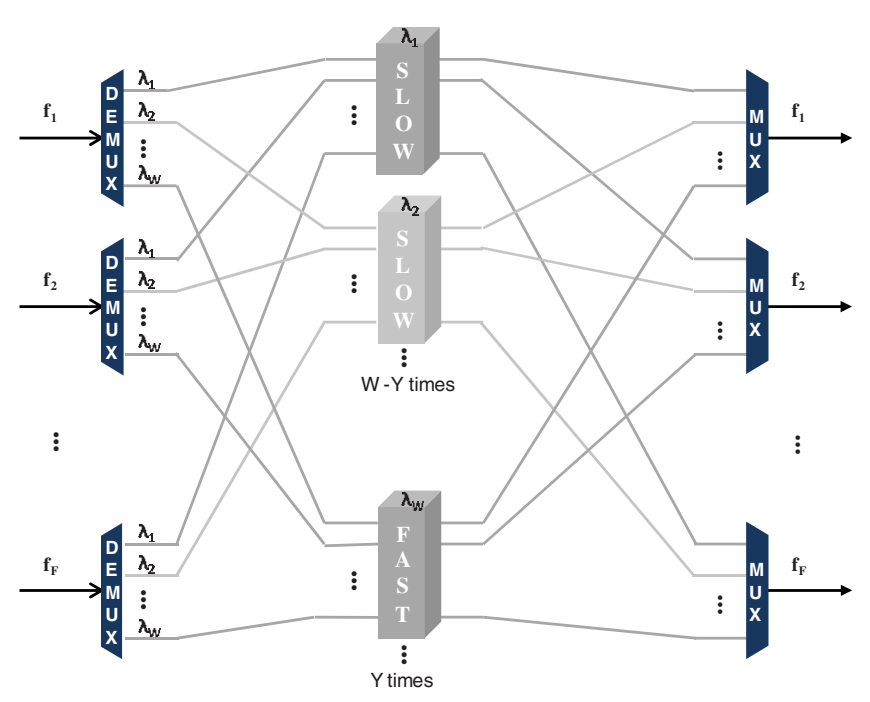

Fig. 2. (Color online) Parallel wavelength modular architecture.

- Lastly, the number of fast switch elements should be limited as much as possible, in order to create a cost-effective design.

An additional design constraint is that neither wavelength conversion nor buffering capabilities are available. It is particularly important to provide a flexible and scalable solution both in switching dimensionality and in bit rate. The architecture is determined by two fundamental design choices and considers the configuration and connectivity of the slow and fast switch fabrics separately. The first choice is related to the slow switch and is based on the welldefined concept of wavelength or link modularity. The former means that identical wavelengths from different fibers are switched in the same fabric, whereas link modularity implies that all wavelengths of a link are grouped in a switching block. The second part of the design introduces the fast switch elements to the multi-granular architecture and is based on the relative connectivity to the slow switch fabric. Possible approaches are either parallel or sequential integration, and this choice has important consequences on the configurability and dimensions of the designs. In Table II, a number of notations are introduced that are relevant for the design and analysis of the MGOXC. Each architecture consists of $F$ input and $F$ output fibers, labeled $f_{1}$ to $f_{F}$. The input fibers are first

TABLE II

Notations For ANAlysis of Dimensions and ScAlability

\begin{tabular}{cl}
\hline Symbol & Meeting \\
\hline$F$ & Number of fibers \\
$W$ & Number of wavelengths per fiber \\
$Y$ & Number of fast wavelengths per fiber \\
$Z_{w}$ & Number of fibers having wavelength $w$ switched \\
& fast \\
\hline
\end{tabular}

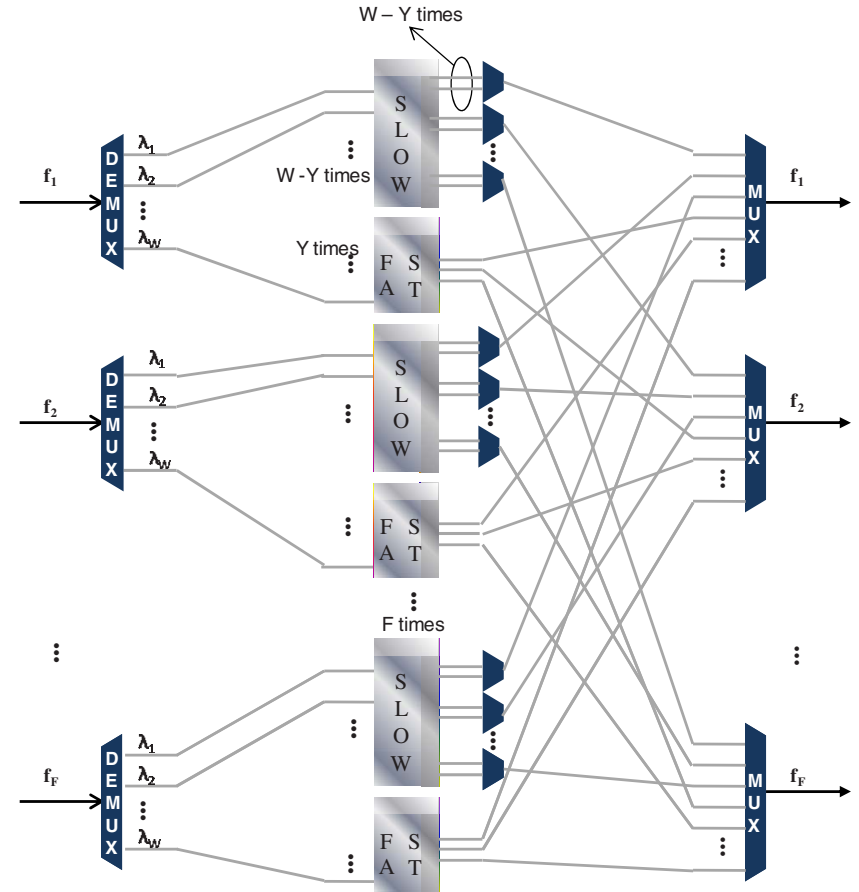

Fig. 3. (Color online) Parallel link modular architecture.

split into different wavelengths by a demultiplexer (DEMUX, by using for example an AWG (arrayed waveguide grating)). Before leaving the OXC, wavelengths are multiplexed (MUX, e.g., AWG) on a fiber. First, the conceptually simple parallel designs (Figs. 2 and 3) provide no connectivity between the slow and fast switch elements, which implies that there is no flexibility to rearrange slow and fast wavelengths, unless it is done manually. In the link modular design, the role of the multiplexing devices (e.g., optical couplers) that are attached to the output of the slow switches, is to ensure the non-blocking behavior as previously defined. The following designs (Figs. 4 and 5) are referred to as the sequential architectures; these provide two-stage switch connectivity for provisioning of fast cross-connections. The fast optical ports can be reached through the slow switches and as such allow (re)configuration of fast connections, shared among all slow switches in order to combine wavelengths from all incoming fibers. Figure 4 is based on the assumption that not only the slow, but also the fast switch is configured in a wavelength modular fashion. However, it is also possible to share a fast switch block between different wavelengths, in order to reduce the number and dimensions of the fast switch fabrics. Also note that for the sequential wavelength modular design, the parameter $K$ is given by $K=\Sigma_{w=1}^{W}\left(1-\delta_{Z_{w} 0}\right)$, where $\delta_{i j}$ is the Kronecker delta, ${ }^{1}$ i.e., $K$ represents the number of distinct wavelengths (total $W$ ) that are switched fast on at least 1 fiber. The sequential link modular design also requires the cou-

\footnotetext{
${ }^{1}$ By definition, the Kronecker delta $\delta_{i j}$ equals 1 if $i=j, 0$ otherwise.
} 


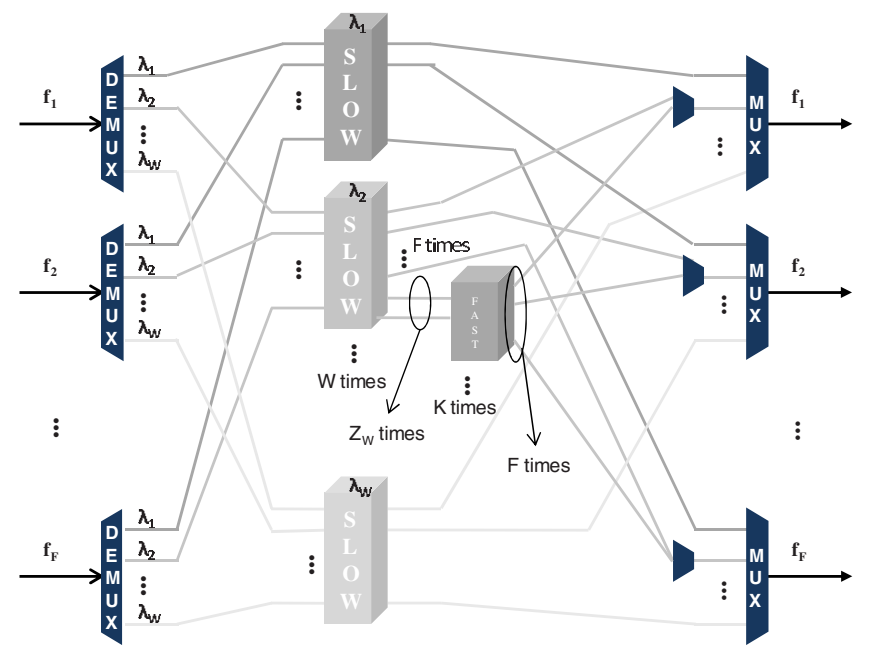

Fig. 4. (Color online) Sequential wavelength modular architecture.

pling devices at the output of the slow switch, in order to adhere to the non-blocking requirement. In Fig. 5, the assumption with regard to the wavelength modularity of the fast switch has been made as well. Again, further reductions in fast switch ports and fabrics are possible by sharing a fast block between different wavelengths.

\section{B. Dimensions and Scalability}

To analyze the dimensions in terms of the number of cross-connects required and the number of ports on each cross-connect, refer to the notations as listed in Table II. The assumption has been made that all fibers support an identical number of fast wavelengths $Y$ :

$$
\sum_{w=1}^{W} Z_{w}=Y \times F .
$$

Also note that it holds that Table III presents the number of slow and fast switches required to realize

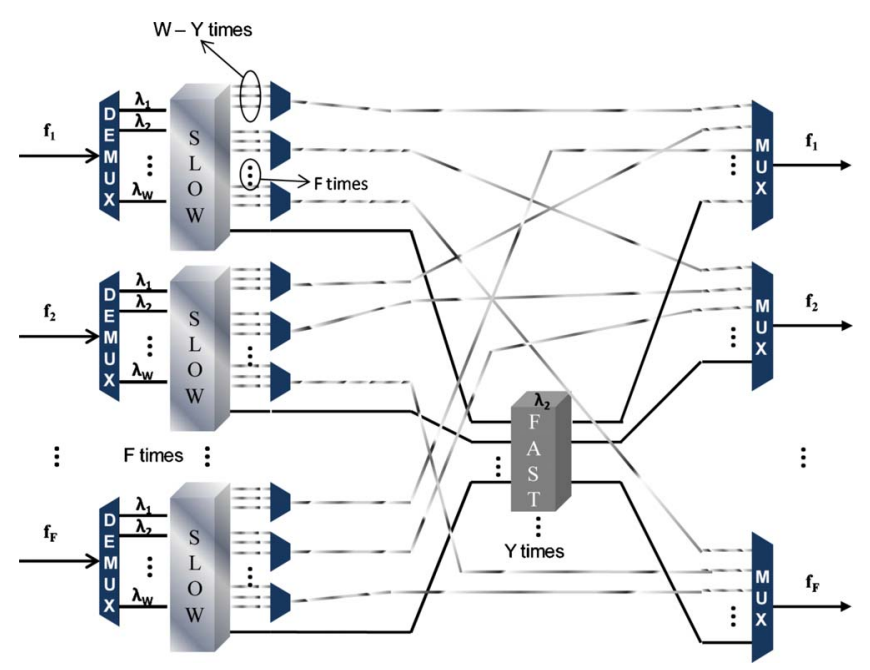

Fig. 5. (Color online) Sequential link modular architecture.
TABLE III

Count and Dimensions of Slow and Fast Switches

\begin{tabular}{clcc}
\hline \multicolumn{1}{c}{ Type } & \multicolumn{1}{c}{ Modularity } & Count & Dimensions \\
\hline \multirow{4}{*}{ Sequential } & Link Slow Switch & \\
& Wavelength & $F$ & $W \times((W-Y) \times F+Y)$ \\
Parallel & Link & $F$ & $F \times F$ \\
& Wavelength & $W-Y$ & $F \times F) \times((W-Y) \times F)$ \\
\multirow{4}{*}{ Sequential } & Link Fast Switch & $Y$ & \\
& Wavelength & $K$ & $F \times F$ \\
Parallel & Link & $F$ & $Z_{w} \times F$ \\
& Wavelength & $Y$ & $F \times(Y \times F)$ \\
& & & $F \times F$ \\
\hline
\end{tabular}

the different architectures, while also indicating their dimensions.

\section{Qualitative Analysis}

In this subsection, we discuss qualitative advantages and disadvantages between the proposed architectures. The focus lies on demonstrating additional features or shortcomings related to the flexibility, reconfigurability, and construction of these switch designs. Since a major design goal of the MG-OXC is cost efficiency, the focus should be on minimizing both the number of fast switching fabrics and ports. Table III does not allow general conclusions to be drawn, as a comparison between the sequential and parallel approaches requires us to make assumptions on the number of fibers $F$, the number of fast wavelengths $Y$, and the number of distinct fast wavelengths $K$. Recall, however, that the sequential designs offer reconfigurability of fast wavelengths, which the parallel architectures lack (see Section III). In Fig. 4, the sequential design assumes identical wavelengths are switched in a single fast block. A further reduction of fast blocks and/or ports is possible by allowing different wavelengths to share a single fast block, as depicted in Fig. 5. In that case, however, the switch's control unit must prevent identical wavelengths from arriving at the MUX of a single fiber. Another possibility is to introduce wavelength conversion inside the MG-OXC, a solution that is currently impractical due to its high cost and technological immaturity.

As previously stated, an important distinction lies in the modularity of the switch designs, which has a major influence on scalability. The link modular approaches easily allow a new link to be added to an existing switch, although extending a link with an extra wavelength is much less straightforward. Likewise, the extensibility of the wavelength modular approaches is straightforward when adding a wavelength, but it is much less convenient to introduce a new link in the switch. 
Depending on integration and/or production costs, a potential advantage can arise for architectures having fast switches, whose input dimensions are smaller than the number of output ports. This is obviously the case for the parallel link modular and sequential wavelength modular approaches.

Finally, the limited scalability of fast switching technologies (e.g., SOA up to $32 \times 32$ ports) may prove an issue when larger fast switch blocks are required. It is, however, possible to combine multiple switch blocks in a multi-stage design as shown in, e.g., $[23,24]$, but careful analysis of physical performance (signal loss, BER, etc.) is advised.

\section{Simulation AnAlysis}

In this section, simulation analysis is used to provide insight in the behavior of the MG-OXC [25]. The implementation allows us to evaluate an MG-OXC in a generic way, independent of architectural details (modularity, reconfigurability). Note that for fixed $F$, $W$, and $Y$ values, the designs presented in the previous section are functionally equivalent. A comparison between MG-OXC and traditional, single-speed OXCs (slow only, fast only) is presented, and results are given for varying traffic load, fractions of slow/fast traffic and number of slow/fast ports available. However, we start with introducing the different approaches to wavelength assignment, which are necessary for mapping incoming data bursts to a suitable wavelength. For a general overview of the node simulations, and to observe the different steps in which traffic is processed, refer to Fig. 6 .

\section{A. Wavelength Assignment}

The introduction of an MG-OXC in a network effectively creates a wavelength partitioning, by grouping wavelengths that are switched on the same type of switching fabrics. As such, an algorithm is required to assign generated traffic to a suitable wavelength partition and the available wavelengths within a partition. This algorithm will be executed at the network's edge, thus before entering the all-optical data trans- port network (see Fig. 6). In the following, the assumption was made that only two partitions (corresponding to slow and fast) are introduced.

As shown in Fig. 7, generated traffic is first classified in slow (arrival rate $\lambda_{s}$ ) and fast $\left(\lambda_{f}\right)$ traffic flows, by inspecting the offset time $T_{\text {offset }}$ between the burst header and the actual data burst. Obviously, for slow traffic it holds that $T_{\text {offset }}>T_{\text {slow }}\left(T_{\text {slow }}\right.$ is the switching speed of the slow switch), while $T_{\text {offset }}<T_{\text {slow }}$ is true for fast traffic $\left(T_{\text {fast }}\right.$ is the switching speed of the fast switch fabric). Based on this classification, a number of alternatives are now possible for assignment of traffic to the wavelength partitions.

The approaches differ in the way traffic is transferred between the slow and fast wavelength partitions. Simple wavelength assignment is the most basic approach, whereby slow bursts are assigned to the slow wavelength partition, and the burst is dropped in case no free wavelength is available. Fast bursts are considered for assignment to the fast wavelength partition in a similar way. The slow-to-fast approach differs from the simple algorithm by allowing slow bursts on the fast wavelengths, only in case these cannot be accommodated on the slow wavelengths. The corresponding fast-to-slow wavelength assignment allows transfer of fast bursts onto slow wavelengths (again only in case the fast burst cannot be assigned to a fast wavelength). We motivate the use of this algorithm as follows: although the slow switch cannot be configured in time for a fast burst, it is possible that the preceding (slow) burst requests the same output, and thus reconfiguration of the switch is not required. Finally, the greedy approach allows transfer of traffic between both wavelength partitions, again only when no available capacity can be found for the original wavelength assignment.

Let $\lambda_{s f}$ be the transfer rate from the slow to the fast wavelength assignment block, and $\lambda_{f s}$ from fast to slow. Then Table IV shows the transfer rates for the different wavelength assignment approaches. Here, $P_{s}$ and $P_{f}$ represent the blocking probabilities of the slow and fast wavelength assignment blocks and are given by (assuming Poisson arrivals)

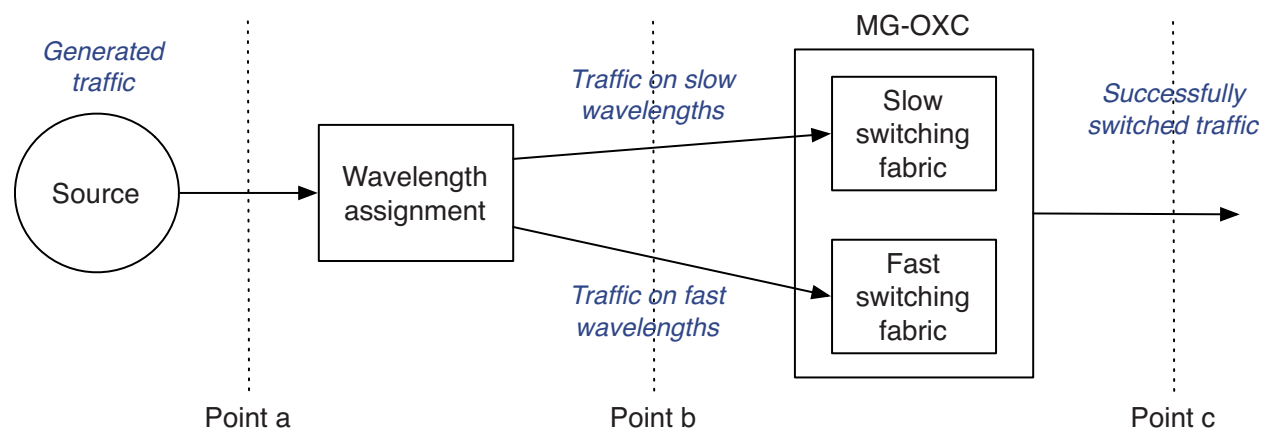

Fig. 6. (Color online) Overview of node simulations. 


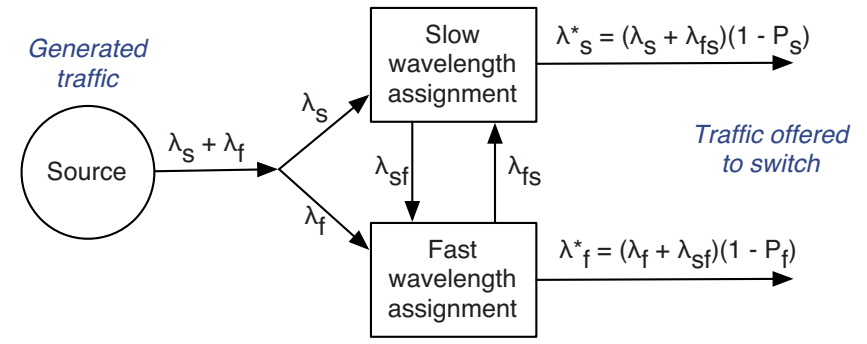

Fig. 7. (Color online) Overview of wavelength assignment.

$$
P_{s}=\operatorname{Erl}\left(\frac{\lambda_{s}+\lambda_{f s}}{B}, W_{s}\right) \text { and } P_{f}=\operatorname{Erl}\left(\frac{\lambda_{f}+\lambda_{s f}}{B}, W_{f}\right)
$$

In these expressions, $B$ represents the bandwidth of a wavelength, $W_{s}$ and $W_{f}$ are the number of slow and fast wavelengths in the slow and fast partitions, respectively, and $\operatorname{Erl}($.$) is the Erlang-B function defined$ as

$$
\operatorname{Erl}(\rho, W)=\frac{\frac{\rho^{W}}{W !}}{\sum_{i=0}^{W} \frac{\rho^{i}}{i !}}
$$

Note that in case of greedy wavelength assignment, $P_{s}$ and $P_{f}$ depend on each other, and thus an iterative substitution is required to obtain the respective blocking probabilities.

To demonstrate the influence of these alternatives, Fig. 8 shows the load remaining after wavelength assignment, i.e., the plot shows the total load at point $b$ for varying generated loads at point a (Fig. 6). This result was obtained by assuming $W_{f}=2$ and $\beta=\lambda_{f} / \lambda_{s}$ $+\lambda_{f}=0.2$, which reflects the expected low number of (expensive) fast wavelengths. It also follows that $\beta$ represents the fraction of generated fast traffic to the total generated (slow and fast) traffic. Even though the total load after wavelength assignment is similar for the various approaches, Fig. 9 provides more insight into which type of traffic is favored. The figure shows the fraction of fast traffic to the total traffic after wavelength assignment, and this for a varying total generated load. In other words, we plot the fraction of fast traffic to the total traffic at point $b$ in Fig. 6 and do this for various load averages at point a. Clearly, the fast-to-slow approach allows more fast traffic than

TABLE IV

TRANSFer RATES (NUMBer OF BURSTS PeR TIME Unit) Between Slow and Fast Wavelength Assignment Blocks

\begin{tabular}{ccccc}
\hline & Simple & Slow-to-fast & Fast-to-slow & Greedy \\
\hline$\lambda_{s f}$ & 0 & $\lambda_{s} P_{s}$ & 0 & $\lambda_{s} P_{s}$ \\
$\lambda_{f s}$ & 0 & 0 & $\lambda_{f} P_{f}$ & $\lambda_{f} P_{f}$ \\
\hline
\end{tabular}

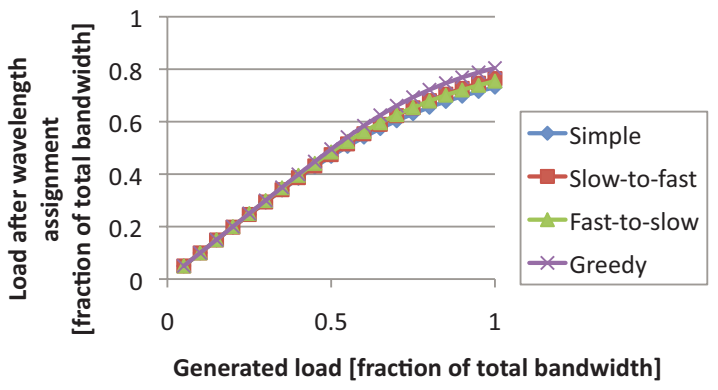

Fig. 8. (Color online) Total load after wavelength assignment is similar for the various algorithms.

the greedy approach, since the latter also allows slow bursts to use valuable fast wavelengths. It should be noted, however, that, although not shown, the behavior of fast-to-slow converges to greedy for increasing values of $\beta$. Both the simple and slow-to-fast algorithms preserve only small fractions of fast traffic and are thus not well-adapted to support a multi-granular optical network scenario, as a non-negligable amount of fast traffic will be lost because of inappropriate wavelength scheduling. Since our main interest is the effect of fast traffic and fast wavelengths, the simulation results presented in Subsection IV.B have been made using the fast-to-slow wavelength assignment algorithm. As mentioned before, another important decision is how bursts are assigned to individual wavelengths within a partition. Strategies such as first-fit or best-fit have previously been investigated in, e.g., [26,27]; however, this subject falls outside the scope of this work. The simulation studies in Subsection IV.B assume a first fit strategy.

\section{B. Single Node Simulations}

This section presents discrete event simulation results of several OXC alternatives (slow only, fast only, and MG-OXC). All designs support 2 input and 2 output fibers, each fiber carrying 10 wavelengths. Neither wavelength conversion nor buffering capability is present in any of the switch designs. Each incoming

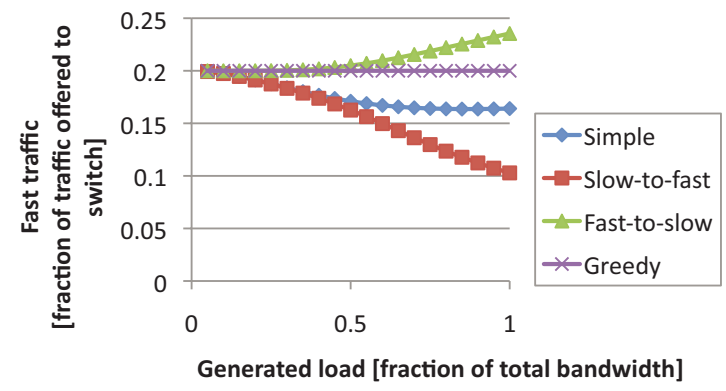

Fig. 9. (Color online) Fraction of fast traffic after wavelength assignment: depending on wavelength assignment, the fraction of traffic sent on fast wavelengths deviates from the generated $\beta$ $=0.2$. 
data burst has a $50 \%$ probability of choosing the first output fiber. The bandwidth of each wavelength is $10 \mathrm{Gbps}$, and traffic is generated according to a Poisson process with an average inter-arrival time of $15 \mathrm{~ms}$. Data sizes follow an exponential distribution, with a varying average to establish the generated load. Because of the limited scale of currently deployed OBS networks, ${ }^{2}$ there is no conclusive data available on a number of relevant traffic parameters. Thus, to control and evaluate the influence of different traffic types, the offset times between the control packet and data are modeled as a 2-phase hyperexponential distribution. The probability density function (pdf) $f$ is given by $f=\alpha \times f_{\text {slow }}+\beta \times f_{\text {fast }}$, with $\alpha+\beta$ $=1$ and $\alpha$ and $\beta$ representing the fractions of generated slow and fast traffic. ${ }^{3}$ The pdf of the slow $f_{\text {slow }}$ (respectively fast $f_{\text {fast }}$ ) traffic is an exponential distribution with average $100 \mathrm{~ms}$ (respectively $10 \mathrm{~ns}$ ). The slow switching fabric has a switching speed of $T_{\text {slow }}$ $=10 \mathrm{~ms}$, while the fast switch has $T_{\text {fast }}=1 \mathrm{~ns}$. These values are representative for a MEMS-based (respectively SOA-based) switch $[5,6]$. This leads to $1-e^{-0.1}$ $=9.5 \%$ of slow traffic that actually belongs to fast traffic, and an identical fraction of generated fast traffic will have $T_{\text {offset }}<T_{\text {fast }}$.

In the following subsections, we show the performance of the MG-OXC switch and compare the results to designs composed of a single switch fabric (slow only, fast only). To allow fair comparison of the results, the wavelength assignment algorithm is also applied in case single-fabric designs are used. This way, the offered traffic pattern at the switches' input ports is identical in all cases. As such, wavelengths are also partitioned in these single-fabric scenarios, even though the switching speeds are identical for all wavelengths. The fast-to-slow wavelength assignment algorithm (Subsection IV.A) was implemented, together with a first-fit approach for mapping data bursts on a specific wavelength within a partition. The results shown focus on the total loss rate of the switch; bursts can be lost either due to contention or because the switching speed is insufficient for a given burst.

1) Varying fraction of fast traffic: In the first experiment, 2 wavelengths are available in the fast partition, while the remaining 8 are allocated for the slow partition. Simulations were performed to evaluate the influence of the fraction of fast traffic for the three switch designs. The resulting Fig. 10 shows the total loss rate (i.e., ratio of dropped traffic to the offered

${ }^{2}$ OBS is still considered an immature technology, and as such OBS testbeds/prototypes are composed of at most a few nodes.

${ }^{3}$ Note that this arrival model does not generate these precise fractions of slow and fast traffic. For bursts generated according to $f_{\text {slow }}$, it is still possible that $T_{\text {offset }}<T_{\text {slow }}$. Using the cumulative distribution function of an exponentially distributed variable, this holds for the following fraction of traffic: $P\left[T_{\text {offset }} \leqslant T_{\text {slow }}\right]=1-e^{-T_{\text {slow }} / T_{\text {offset }} \text {. The }}$ same argument holds for fast traffic, where $T_{\text {offset }}<T_{\text {fast }}$.

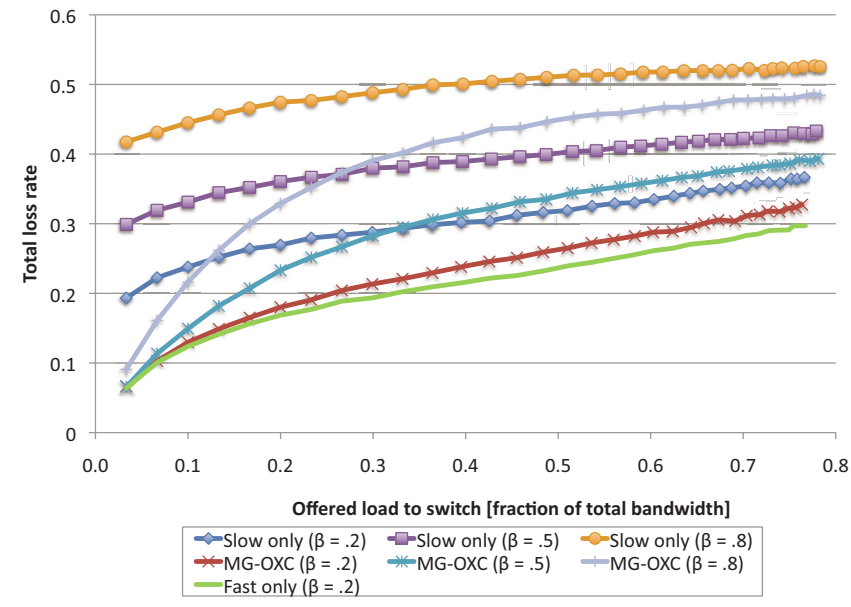

Fig. 10. (Color online) Higher fractions of fast traffic increase the total loss rate for a fixed number of fast wavelengths $\left(W_{f}=2\right)$.

load measured at point $\mathrm{c}$ in Fig. 6) for a varying offered load (point b in Fig. 6). First observe that, for low loads, the relatively high loss rates can be attributed to the fraction of fast traffic that has an offset time lower than the fast switching speed. However, some of these bursts can still be switched correctly as consutive bursts taking the same output port does not require reconfiguration of the switch fabric (this explains the loss rate close to $6.5 \%$ of the fast only design in comparison to the predicted 9.5\%).

Then, an increasing fraction $\beta$ of fast traffic causes higher loss rates, since the number of fast switching ports remains fixed ( 0 for the slow only, 2 for the MG$\mathrm{OXC}$ ). This does not apply to the fast only design (only shown for $\beta=0.2$ ), whose performance is very similar for all fractions of fast traffic. Also, it is readily apparent that the MG-OXC outperforms the slow only design for all values of $\beta$. Another observation is that the MG-OXC offers loss rates similar to the fast only design, unless high fractions of fast traffic are generated ( $\beta=0.5$ and 0.8 ). This is not surprising considering the small number of fast switching ports available to the MG-OXC.

2) Varying number of fast wavelengths: In the following experiment, the generated traffic consisted of $80 \%$ fast traffic $(\beta=0.8)$. Now, simulations focus on varying the number of slow/fast wavelengths in each partition and hence also the exact number of slow/fast wavelengths available to the MG-OXC. Figure 11 shows the total loss rate (point c in Fig. 6) for a varying offered load (point $b$ in the same figure), where one can immediately observe that an increased number of fast wavelengths results in a lower loss rate. That this result holds even for the slow only designs is due to the use of the fast-to-slow wavelength assignment and the simulation setup: the initial switch configuration connects the top input and output fibers (and likewise for the bottom fibers), and traffic is generated with a 


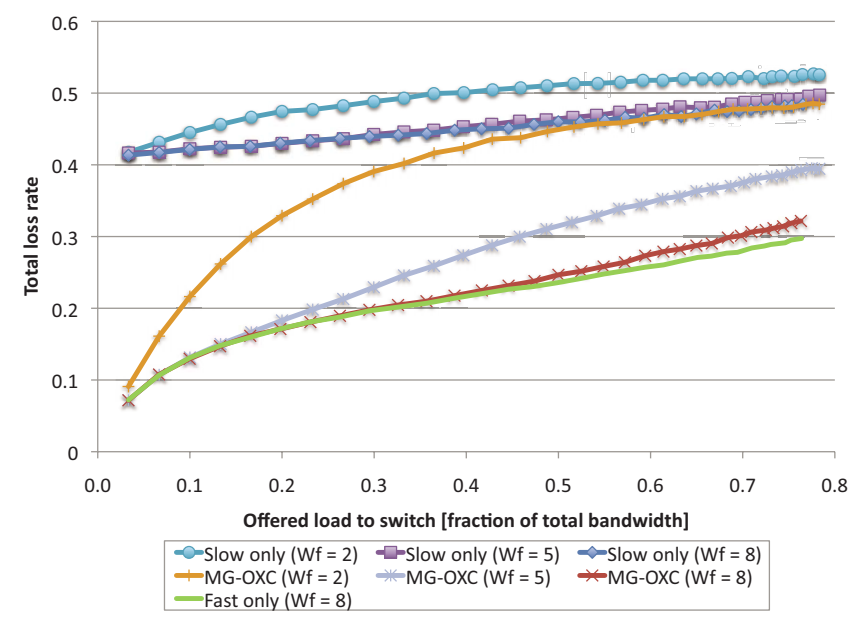

Fig. 11. (Color online) Higher number of fast wavelengths decrease the total loss rate for a fixed fraction of fast traffic $(\beta=0.8)$.

$50 \%$ probability of choosing either output fiber. Consequently, more or less half of the traffic on the $W_{f}$ wavelengths can be switched correctly, and this explains why increasing values of $W_{f}$ reduce the total loss rate. As before, the MG-OXC can provide an overall improved loss performance compared to the slow only design (behavior of slow only and MG-OXC are similar only for high loads and a severely under-dimensioned fast switching block). For high numbers of fast wavelengths $\left(W_{f}=8\right)$, the loss rate of the MG-OXC approaches the performance of the fast only design. Note again that results of the fast only design are shown only for $W_{f}=8$, as other values for $W_{s}$ lead to very similar loss rates. A final observation is that, although not shown, the loss rate of the slow only design is slightly higher in case greedy wavelength assignment is used, due to an assignment of fast bursts to slow wavelengths.

In conclusion, this section clearly demonstrated that an MG-OXC, equipped with only a limited amount of fast ports, can offer significant improvements in loss rates when compared to a slow only design. Furthermore, as long as the mismatch between fast traffic and fast wavelengths remains within acceptable bounds, the MG-OXC can approach the performance levels offered by a fast only design.

\section{Demonstration of MG-OXC ARChiteCtures IN A HYBRID NETWORK TESTBED}

The functionality and performance of the multigranular switching concept has been evaluated and experimentally demonstrated on an application-aware multi-bit-rate OBS testbed at University of Essex [28]. The application-aware core OBS router utilizes a PowerPC processor embedded in an FPGA and a MGOXC composed of a fast and widely tunable SG-DBR (sample grating distributed Bragg reflector) laser, a
2D MEMS switch, and a fast SOA-MZI switch. The multi-granular switch fabric is wavelength selective and is controlled by extraction and processing of the incoming $\mathrm{BCH}$ through a clock and data recovery (CDR) and a word alignment unit (WAU). Then the header processing block (HP) is used to retrieve all the required information from the $\mathrm{BCH}$ and the header re-insertion (HRI) unit is used to re-insert the $\mathrm{BCH}$ on the control plane lambda. The BCH consists of several fields as illustrated in Table V. The class of service field (CoS) explicitly determines the latency requirements of the burst and thus selects the appropriate switch fabric. The offset time together with the burst length, wavelength (Burst $\lambda$ ), source (Src) and destination (Dest) provide detailed configuration parameters for either the slow or fast switch fabric. The FPGA provides the appropriate electronic control signals for the MEMS switch state and the tunable laser that optically controls the SOA-MZI. The MG-OXC comprises a low cost, commercial, high-dimension, slow 2D MEMS switch $(10 \mathrm{~ms})$ and a high cost, lowdimension but fast (1 ns) SOA-MZI-based switch. Figure 12(a) illustrates the functional blocks of the testbed, including the parallel [Fig. 12(b)], and sequential [Fig. 12(c)] switch architectures. The egress node detects incoming bursts and can measure the BER of individual bursts, based on the appropriate control (gating) signals generated by an FPGA. The node processor integrates a CDR mechanism, a WAU, as well as a header processing (HP) unit. When an incoming $\mathrm{BCH}$ is received, the HP unit extracts the header length, offset, and burst length and provides a signal (envelope) used to gate the BER test equipment for the duration of the received burst. Finally, the control plane is based on just in time (JIT) signaling and operates out-of-band in a full duplex mode at 2.5 Gbps.

\section{A. Control and Data Plane Implementation}

Figure 13 shows the testbed architecture and experimental setup. The OBS testbed operates at 2.5 Gbps for the control plane and 10 and 40 Gbps for the data plane. All routers utilize a Xilinx high-speed and high-density VirtexII-Pro FPGA with an embedded PowerPC processor. The OBS control channel is full duplex (to allow synchronization) for both links and is transmitted on dedicated wavelength channels using the proprietary Optical Burst Ethernet Switched (OBES) transport protocol [29]. The data plane transports variable sized bursts with variable

TABLE V

Burst Control Header (BCH) Format

\begin{tabular}{|c|c|c|c|c|c|c|c|}
\hline Burst & Header & $\mathrm{CoS}$ & Burst & Offset & Burst & Src. & Dest. \\
\hline ID & Length & & Length & & $\lambda$ & & \\
\hline
\end{tabular}




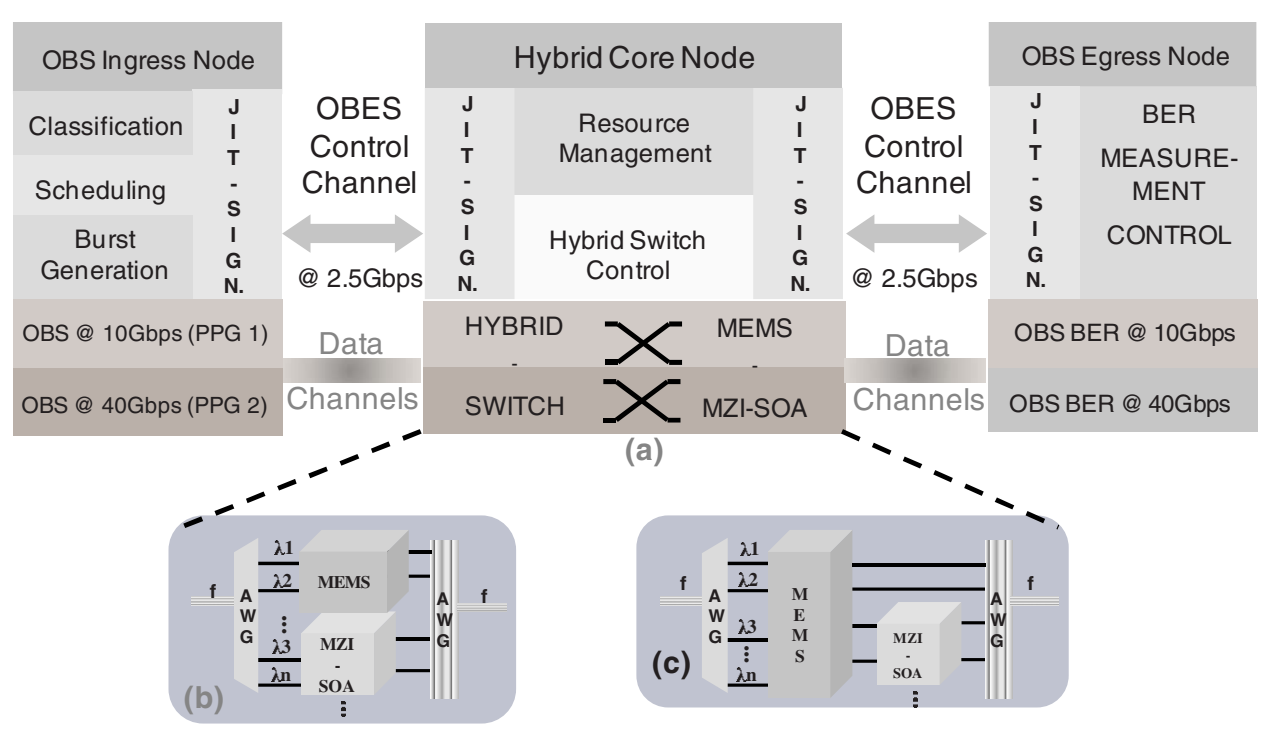

Fig. 12. (Color online) (a) Multi-bit-rate OBS testbed to demonstrate and evaluate different MG-OXC architectures: (b) parallel, (c) sequential.

time intervals on burst mode. The electronic section of the ingress edge router incorporates a differentiation scheduler (DS), classification scheduler (CS), wavelength allocation scheduler (WAS), and $\mathrm{BCH}$ assembly and assignment. Burst generation is performed by controlling two $10 \mathrm{Gbps}$ PPGs that generate the emulated bursts carrying $2^{7}-1$ pseudo random binary sequences (PRBS). The control signals for the bursts are envelope patterns with variable duration and time intervals and are generated via the Rocket IO interface of the FPGA to gate the PPGs. The output from one of the two PPGs is used to externally modulate an optical carrier at $\lambda_{5}=1553.00 \mathrm{~nm}$ from a DFB laser using a Mach-Zehnder modulator (MZM) producing the 10 Gbps NRZ burst data stream. The second PPG modulates a $10 \mathrm{GHz}$ optical pulse stream (1.4 ps FWHM, sech ${ }^{2}$-shaped pulse) from a tunable modelocked laser (TMML) at $\lambda_{6}=1556.65 \mathrm{~nm}$, generating a 10 Gbps RZ burst data stream. Further, the bursts are time-multiplexed to $40 \mathrm{Gbps}$ through a two-stage optical multiplexer (OMUX) that maintains the $2^{7}-1$ randomness. At the output of the ingress node, the 10 and 40 Gbps burst data streams are coupled and transmitted over a single fiber. In parallel to the data plane, the edge router generates and optically transmits the $\mathrm{BCH}$ at $2.5 \mathrm{Gbps}$ on an out-of-band wavelength $\left(\lambda_{2}=1543 \mathrm{~nm}\right)$ towards the core router controller. A second out-of-band control channel at $\lambda_{1}$ $=1544 \mathrm{~nm}$ is generated by the core router and received at the ingress node to establish the full duplex control operation. A delay element inside the FPGA at the ingress node is used to synchronize the data plane and control plane, by providing the appropriate offset time between the $\mathrm{BCH}$ and data burst. The actual value of the offset time is based on known propagation delays in the system and processing delays in the core router.

The core router integrates an FPGA, a MEMS switch, a widely tunable SG-DBR laser, and a fast SOA-MZI switch. The FPGA receives and processes the information from the control channel and in turn controls the tunable laser and both switch fabrics. More specifically, the fabric is controlled by means of extracting and processing the incoming $\mathrm{BCH}$ through

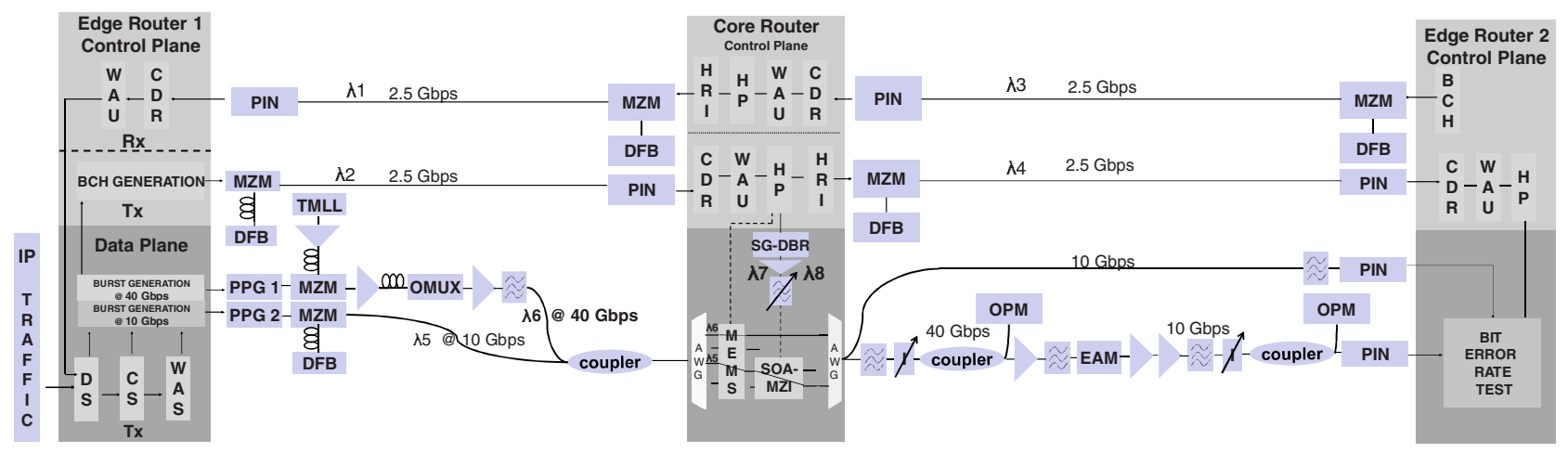

Fig. 13. (Color online) Physical layer representation of application-aware, multi-granular, multi-bit-rate OBS testbed. 
CDR, a WAU, as well as an HP unit. It should be noted that the switch is dynamically configured by the FPGA controller, which performs on-the-fly processing of the incoming $\mathrm{BCH}$ header information. According to CoS, offset, burst length, wavelength, and destination, the FPGA provides the appropriate electronic control signals for the MEMS switch state (for slow switching), and the appropriate tuning sequences for the tunable laser that optically controls and configures the SOA-MZI (for fast switching). The MEMS switch is configured to have some soft-permanent dedicated cross-connections in order to direct the small and medium sized bursts towards the SOA-MZI [see Figs. $12(\mathrm{~b})$ and 12(c)]. At the SOA-MZI switch, fast switching of the shorter bursts between the bar and crossbar state of the switch is achieved based on the existence of a control wavelength from the tunable laser and the temporal position of this control. The SOAMZI operation is based on standard switching interference when a wavelength is applied to one of the two SOA modules. Finally, the controller reinserts a new $\mathrm{BCH}$ for the next node. The combination of slow and fast switch is wavelength selective and can effectively support OCS, OBS, or even OPS.

Finally at the egress side, the node integratessimilar to the core router-the CDR, WAU, and HP units. The control plane is realized for duplex operation (between the core router and edge router 2) with out-of-band control channels at $\lambda_{3}=1541.3 \mathrm{~nm}$ and $\lambda_{4}$ $=1547.4 \mathrm{~nm}$ for the two directions, respectively. After the arrival of a $\mathrm{BCH}$ on the control channel, the extracted (by the HP unit) information about the offset and the burst length is processed and the appropriate burst envelope is generated and fed to the BER tester for direct BER measurements on the burst level. On the data plane, the $10 \mathrm{Gbps}$ burst data are detected with a pre-amplified receiver, while the $40 \mathrm{Gbps}$ bursts are first demultiplexed to $10 \mathrm{Gbps}$ by means of an electro-absorption modulator (EAM) and then passed to the pre-amplified receiver. In practice, a slightly shorter envelope of the exact burst length is used to gate the BER tester, in order to ease synchronization between the BER tester and the received burst.

\section{B. Experimental Results}

For the experimental evaluation of the MG-OXC, the OBS testbed described above is used to demonstrate dynamic controlled switching of variable-length optical bursts (from $200 \mathrm{~ns}$ up to $30 \mathrm{~ms}$ ) transmitted at $10 \mathrm{Gbps}$ over $\lambda_{5}=1553.00 \mathrm{~nm}$ and $40 \mathrm{Gbps}$ over $\lambda_{6}$ $=1556.65 \mathrm{~nm}$. The 40 Gbps data stream carries smalland medium-sized bursts (200 ns, $3.6 \mu \mathrm{s}, 5 \mu \mathrm{s}$ ), which are passed through the pre-configured MEMS to the SOA-MZI for fast switching. The $10 \mathrm{Gbps}$ data stream can be considered as long bursts or short circuits
(30 ms) and is switched by the MEMS. In parallel to these data bursts, the appropriate $\mathrm{BCH}$ is generated by the ingress node and transmitted to the core router, which in turn controls the MEMS and the SOA-MZI (via the tunable laser). Figure 14(b) shows the $3.6 \mu \mathrm{s}$ long control signal on $\lambda_{7}=1538.94 \mathrm{~nm}$ from the tunable laser that controls the SOA-MZI, and the resulting switched bursts at both output ports for the crossbar switching state. Another burst is switched using a $5 \mu \mathrm{s}$ long control signal on $\lambda_{8}=1542.17 \mathrm{~nm}$ [Fig. 14(c)]. Figure 14(d) shows the $200 \mathrm{~ns}$ control signal on $\lambda_{9}=1548 \mathrm{~nm}$ and the switched bursts on both output ports. The SOA-MZI gate is biased at the saturated point and configured to achieve the maximum static extinction ratio between the two output ports at an operating current of 354 and $360 \mathrm{~mA}$ for both SOAs, respectively. The configuration is based on a counterpropagating set-up between the data and the control signals while a holding beam is used (co-propagating with data) in order to reduce the noise output of the SOAs and improve the functionality of the gate for bursty switching.

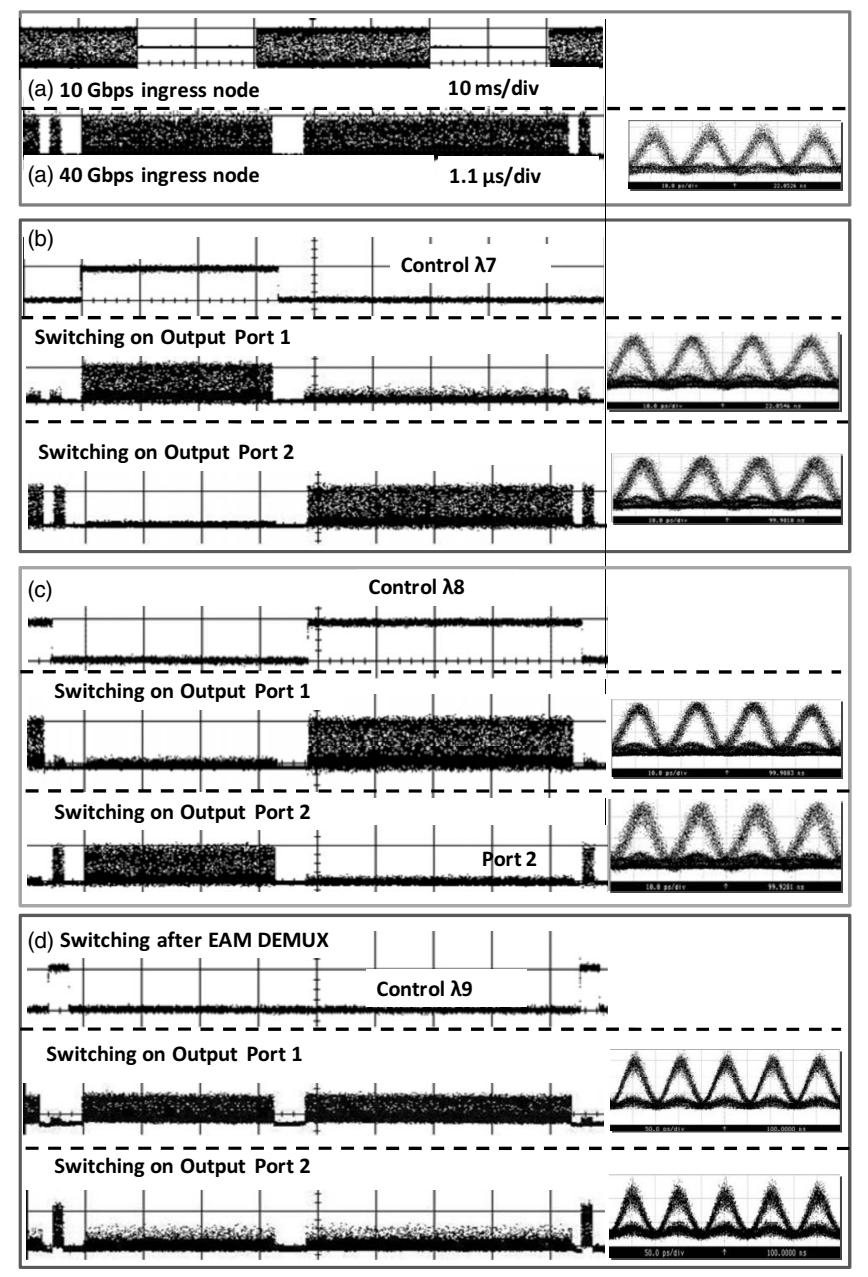

Fig. 14. (a) Variable sized bursts at 10 and $40 \mathrm{Gbps}$, (b) $40 \mathrm{Gbps}$ SOA-MZI burst switching on both output ports based on control $\lambda_{7}$, (c) control $\lambda_{8}$, and (d) control $\lambda_{9}$. 
BER measurements of the fast switched bursts at $40 \mathrm{Gbps}$ through the controllable SOA-MZI switch have been obtained on the burst level after rate demultiplexing with an EAM. The results (Fig. 15) show an acceptable penalty for this type of fast switch that varies between 1 and $2.2 \mathrm{~dB}$ for all the studied cases; these are the four possible bar and cross-bar switching states and for both control signals at $\lambda_{7}$ and $\lambda_{8}$. BER measurements at the output port of the MEMS switch revealed a negligible penalty for both the 10 and 40 Gbps burst data streams, implying that signal quality is not affected by the slow switch fabric. So the overall performance of the MG-OXC is independent of the relative connectivity of the fast switch element (SOA-MZI) to the MEMS switch element. So both sequential and parallel MG-OXC architectures have the same physical layer performance.

It is also noteworthy that the utilized SOA-MZIbased set-up for fast switching is polarization sensitive. The optimization of polarization controllers and the dynamic extinction ratio of the switch is done only once for the control signal on $\lambda_{7}$ at switching port 2 and maintained for all other cases. This is the reason why this particular case shows better BER performance ( $1 \mathrm{~dB}$ penalty) compared to the other three cases (up to $2.2 \mathrm{~dB}$ penalty).

In this experiment, the main purpose was to successfully demonstrate the functionality of a multigranular switch able to operate at multiple bit rates in an application-aware mode, defined by the appropriate control messages generated at the ingress node and processed at the core and egress router. For this reason, a simple type of fast switch has been chosen with certain known limitations. However, the intrinsic operating flexibility of the multi-granular OBS set-up presented here, as well as the flexibility in the node architecture, allows the implementation of different

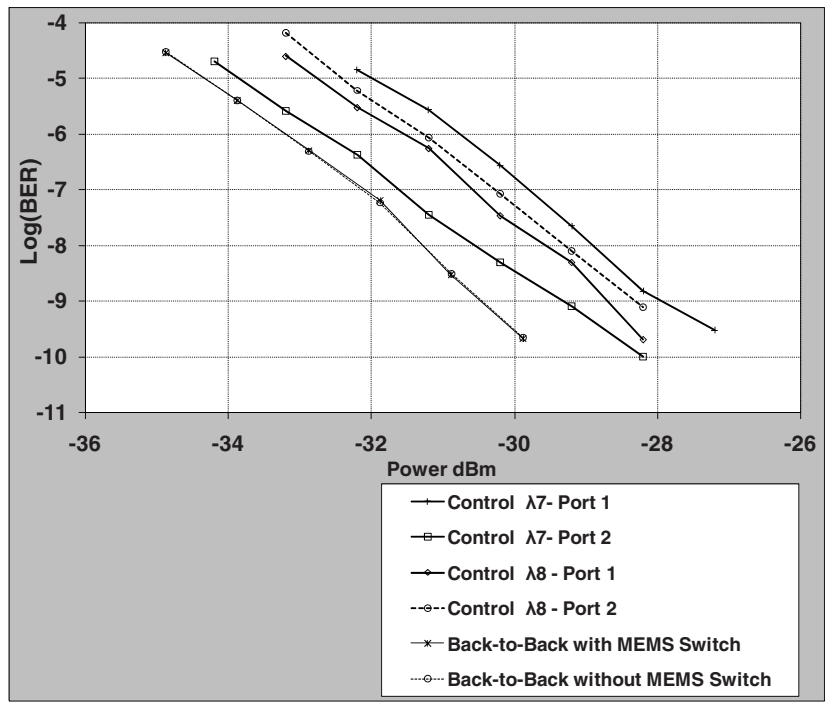

Fig. 15. BER performance of the multi-granular switch. types of fast switching set-ups, as the control signals are generated from the core router processor and can be easily adjusted to any switching type. In this sense, polarization insensitive switching types [30,31] or even regenerative switching schemes [32,33] can be applied, offering superior performance in terms of BER and better stability almost independent of the input signals. Additionally, the use of the fast tunable laser-based controller at the core router allows the adoption of wavelength conversion schemes either for switching (through passive routing via AWGs) or for contention resolution purposes [33]. The utilized tunable laser is capable of switching between 85 different wavelengths in less than $100 \mathrm{~ns}$ and with a maximum peak power difference of only $0.5 \mathrm{~dB}$.

\section{CONCLUSIONS}

Existing and emerging applications require dynamic optical networks able to support different traffic profiles with different levels of QoS (e.g., latency, loss). Simultaneously, technologies like OCS, OBS, and OPS seek flexible and agile combinations of wavelength and sub-wavelength granularity for switching and routing of circuits, packets, and bursts directly in the optical layer. MG-OXC is able to realize all mentioned functionality by employing a flexible and scalable combination of relatively simple, low-cost, and high-dimension optical switching fabrics with millisecond switching speeds and low-dimension and expensive switch elements with fast switching speeds. This paper presented the design and node-level analysis of MG-OXCs. Furthermore, simulation analysis was used to evaluate a number of important parameters concerning traffic load and switch design. Results indicate that a MG-OXC with a limited number of fast ports has similar performance to a design that consist solely of fast ports. Finally, we demonstrated the feasibility of this architecture on an applicationaware multi-bit-rate end-to-end OBS testbed. The deployed hardware consisted of a low-cost, commercial, high-dimension, slow 2D MEMS switch $(10 \mathrm{~ms})$ and a high-cost, low-dimension but fast (1 ns) SOA-MZI based switch.

\section{ACKNOWLEDGMENT}

The work described in this paper was carried out with the support of the BONE-project ("Building the Future Optical Network in Europe"), a Network of Excellence funded by the European Commission through the 7th ICT-Framework Programme as well as a bilateral project funded by GSRT, Greece, and the British council called Autonomous Optical Networks for Global Grid Computing Applications. M. De Leenheer is funded by the IWT through a Ph.D grant, and C. 
Develder is supported by the FWO through a post-doc grant.

\section{REFERENCES}

[1] D. Simeonidou, R. Nejabati, G. Zervas, D. Klonidis, A. Tzanakaki, and M. J. O'Mahony, "Dynamic optical network architectures and technologies for existing and emerging grid services," J. Lightwave Technol., vol. 23, no. 10, pp. 3347-3357, Oct. 2005.

[2] M. J. O’Mahony, D. Simeonidou, D. K. Hunter, and A. Tzanakaki, "The application of optical packet switching in future communication networks," IEEE Commun. Mag., vol. 39, no. 3, pp. 128-135, Mar. 2001.

[3] C. Qiao and M. Yoo, "Optical burst switching (OBS)—a new paradigm for an optical Internet," J. High Speed Networks, vol. 8, no. 1, pp. 69-84, 1999.

[4] C. Qiao, "Labeled optical burst switching for IP-over-WDM integration," IEEE Commun. Mag., vol. 38, no. 9, pp. 104-114, Sept. 2000.

[5] S. J. B. Yoo, "Optical packet and burst switching technologies for the future photonic Internet," J. Lightwave Technol., vol. 24, no. 12, pp. 4468-4492, Dec. 2006.

[6] G. I. Papadimitriou, C. Papazoglou, and A. S. Pomportsis, "Optical switching: switch fabrics, techniques, and architectures," J. Lightwave Technol., vol. 21, no. 2, pp. 384-405, Feb. 2003.

[7] I. Baldine, M. Cassada, A. Bragg, G. Karmous-Edwards, and D. Stevenson, "Just-in-time optical burst switching implementation in the ATDnet all-optical networking testbed," in Proc. IEEE Globecom, San Francisco, CA, USA, vol. 5, pp. 27772781, Dec. 2003.

[8] A. Sahara, Y. Tsukishima, K. Shimano, M. Koga, K. Mori, Y. Sakai, Y. Ishii, and M. Kawai, "Demonstration of optical burst switching network utilizing PLC and MEMS switches with GMPLS control," in Proc. European Conf. Optical Communication (ECOC), Stockholm, Sweden, pp. 896-897, Sept. 2004.

[9] K. Kitayama, M. Koga, H. Morikawa, S. Hara, and M. Kawai, "Optical burst switching network testbed in Japan," in Proc. Optical Fiber Communication Conf. (OFC), Anaheim, USA, Mar. 2005, paper OFA6.

[10] Y. Sun, T. Hashiguchi, V. Q. Minh, X. Wang, H. Morikawa, and T. Aoyama, "A burst-switched photonic network testbed: its architecture, protocols and experiments," IEICE Trans. Commun., vol. E88-B, no. 10, pp. 3864-3873, 2005.

[11] W. Wei, Q. Zeng, O. Y. Yong, and D. Lomone, "Highperformance hybrid-switching optical router for IP over WDM integration," Photonic Network Commun., vol. 9, no. 2, pp. 139-155, Mar. 2005.

[12] A. A. Amin, K. Shimizu, M. Takenaka, R. Inohara, K. Nishimura, Y. Horiuchi, M. Usami, Y. Takita, Y. Kai, Y. Aoki, H. Onaka, T. Miyahara, T. Hatta, K. Motoshima, and Y. Nakano, "Label-based path switching and error-free forwarding in a prototype optical burst switching node using a fast $4 \times 4$ optical switch and shared wavelength conversion," in Proc. Optical Fiber Communication Conf. (OFC), Anaheim, CA, USA, Mar. 2006, Paper OFO5.

[13] R. Takahashi, T. Nakahara, K. Takahata, H. Takenouchi, T. Yasui, N. Kondo, and H. Suzuki, "Photonic random access memory for 40-Gb/s 16-b burst optical packets," IEEE Photon. Technol. Lett., vol. 16, no. 4, pp. 1185-1187, Apr. 2004.

[14] http://www.glimmerglass.com/.

[15] http://www.calient.net/index.php.

[16] X. Cao, Y. Xiong, V. Anand, and C. Qiao, "Wavelength band switching in multi-granular all-optical networks," Proc. SPIE, vol. 4874, pp. 198-210, July 2002.

[17] P. H. Ho and H. T. Mouftah, "Routing and wavelength assignment with multi-granularity traffic in optical networks," $J$. Lightwave Technol., vol. 20, no. 8, pp. 1292-1303, Aug. 2002.
[18] C. T. Politi, C. Matrakidis, A. Stavdas, D. Gavalas, and M. J. O'Mahony, "Single layer multigranular OXCs architecture with conversion capability and enhanced flexibility," J. Opt. Netw., vol. 5, no. 12, pp. 1002-1012, Dec. 2006.

[19] L. Noirie, M. Vigoureux, and E. Dotaro, "Impact of intermediate grouping on the dimensioning of multi-granularity optical networks," in Proc. Optical Fiber Communication Conf. (OFC), Anaheim, CA, Mar. 2001, paper TuG3.

[20] L. Noirie, C. Blaizot, and E. Dotaro, "Multi-granularity optical crossconnect," in Proc. European Conf. Optical Communications (ECOC), Munich, Germany, Sept. 2000, paper 9.2.4.

[21] L. Noirie, F. Dorgeuille, and A. Bisson, " $32 \times 10$ gbit/s DWDM metropolitan network demonstration with 10 waveband-ADMs and $155 \mathrm{~km}$ teralight metro fiber," in Proc. Optical Fiber Communication Conf. $(O F C)$, Anaheim, CA, USA, Mar. 2002, paper ThH4.

[22] M. De Leenheer, C. Develder, B. Dhoedt, and P. Demeester, "Dimensioning of multi-granular optical networks," in Proc. 34th Conf. Optical Communication (ECOC), Sept. 2008, paper P.5.14.

[23] C. Clos, "A study of non-blocking switching networks," Bell System Techn. J., vol. 32, no. 5, pp. 406-424, Mar. 1953.

[24] R. A. Thompson and D. K. Hunter, "Elementary photonic switching modules in three divisions," IEEE J. Sel. Areas Commun., vol. 14, no. 2, pp. 362-373, Feb. 1996.

[25] M. De Leenheer, C. Develder, J. Vermeir, J. Buysse, F. De Turck, B. Dhoedt, and P. Demeester, "Performance analysis of a hybrid optical switch," in Proc. 12th Conf. Optical Network Design and Modelling (ONDM), Mar. 2008.

[26] H. A. Choi and E. J. Harder, "On wavelength assignment in WDM optical networks," Parallel Computing Using Optical Interconnections, vol. 468, pp. 117-136, 1998.

[27] X. Sun, Y. Li, I. Lambadaris, and Y. Q. Zhao, "Performance analysis of first-fit wavelength assignment algorithm in optical networks," in Proc. 7th Int. Conf. on Telecommunications, vol. 2, pp. 403-409, Zagreb, Croatia, June 2003.

[28] G. Zervas, L. Sadeghioon, D. Klonidis, Y. Qin, R. Nejabati, and D. Simeonidou, "Demonstration of novel multi-granular switch architecture on an application-aware end-to-end multi-bit rate OBS network testbed," in 33rd European Conf. Optical Communication (ECOC 2007), PostDeadline, PDS 3.2, Berlin, Germany, Sept. 2007.

[29] G. Zervas, R. Nejabati, D. Simeonidou, and M. J. O'Mahony, "QoS-aware ingress optical grid user network interface: highspeed ingress OBS node design and implementation," in Proc. Optical Fiber Communication Conf. (OFC), Anaheim, CA, USA, Mar. 2006, paper OWQ4.

[30] A. A. Amin, K. Shimizu, M. Takenaka, R. Inohara, K. Nishimura, Y. Horiuchi, M. Usami, Y. Takita, Y. Kai, Y. Aoki, H. Onaka, T. Miyahara, T. Hatta, K. Motoshima, and Y. Nakano, "Label-based path switching and error-free forwarding in a prototype optical burst switching node using a fast $4 \times 4$ optical switch and shared wavelength conversion," in Proc. Optical Fiber Communication Conf. (OFC), Anaheim, CA, USA, Mar. 2006, paper OFO5.

[31] K. Nashimoto, N. Tanaka, M. LaBuda, D. Ritums, J. Dawley, M. Raj, D. Kudzuma, and T. Vo, "High-speed PLZT optical switches for burst and packet switching," in Proc. 2nd Int. Conf. Broadband Networks (IEEE Broadnets), Boston, MA, USA, Oct. 2005, vol. 2, pp. 1118-1123.

[32] C. T. Politi, A. Tzanakaki, D. Klonidis, M. J. O'Mahony, and I. Tomkos, "Optical cross-connect architecture using waveband conversion and a passive wavelength router," Proc. IEE Optoelectronics, vol. 152, no. 4, pp. 215-221, Aug. 2005.

[33] D. Klonidis, C. Politi, R. Nejabati, M. J. O'Mahony, and D. Simeonidou, "OPSnet: design and demonstration of an asynchronous high speed optical packet switch," J. Lightwave Technol., vol. 23, no. 10, pp. 2914-2925, Oct. 2005. 


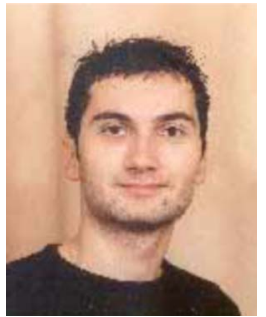

Georgios S. Zervas was awarded the M.Eng. degree in electronic and telecommunication systems engineering with distinction from the University of Essex (UK). He is currently working towards the Ph.D. degree in optical networks for grid application and services at the University of Essex. He is a Senior Research Officer in the Photonic Networks Lab at the University of Essex involved in the EC funded projects MUFINS, e-Photon/ONe+, Phosphorus, and BONE. $\mathrm{He}$ is the author and co-author of over 40 papers in international journals and conferences. His research interests include high-speed optoelectronic router design, optical burst switched networks, GMPLS networks, and grid networks. He is also involved in standardization activities in the Open Grid Forum (OGF) through the Grid High Performance Networking Research Group (GHPN-RG) and the Network Service Interface Working Group (NSI-WG).

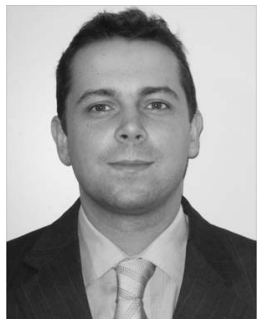

Marc De Leenheer received the M.Sc. degree and $\mathrm{Ph} . \mathrm{D}$. in computer science engineering from Ghent University, Belgium, in June 2003 and Dec. 2008, respectively. He is currently a Post-Doctoral Researcher at the same institution. His main interests include modeling and optimization of grid management architectures, specifically in the context of photonic networks. He is involved in multiple national and European research projects (IST Phosphorus, IST e-Photon/ONe+, IST BONE). He is the author or co-author of over 40 publications in peer-reviewed journals or international conference proceedings.

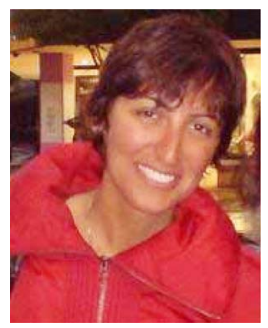

Lida Sadeghioon received the B.S degree in electronic system engineering from Tehran Azad University (Iran) in 1998 and the M.Sc. degree in computer and information networks from the University of Essex (UK) in 2005 . From 1999 to 2004 she was a Network Design Engineer for the Telecommunication Company of Tehran. From 2005 to 2007 she was a Research Officer for the European funded project MUFINS. She is currently perusing a Ph.D. degree on all optical signal processing for high speed optical networks at the University of Essex. Her research interest includes optical signal processing, WDM optical networks, and resource allocation and routing protocols for optical networks.

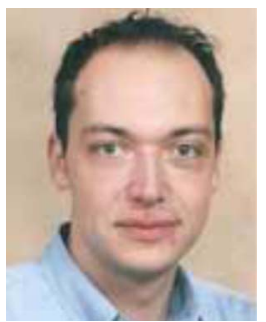

Dimitris Klonidis is an Assistant Professor at Athens Information Technology Centre, Greece. He was awarded his Ph.D. degree in the field of optical communications and networking from the University of Essex, UK, in 2006. In September 2005, he joined the high-speed Networks and Optical Communications (NOC) group in AIT as a faculty member and Senior Researcher. Dr. Klonidis has several years of research and development experience, working on a large number of national and European projects in the field of optical switching, networking, and transmission. He has more than 50 publications in international journals and refereed major conferences. His main research interests are in the area of optical communication networks, including optical transmission and modulation, signal processing and equalization, and fast switching and node control. The considered networking applications include future access networks, and optical packet/burst switched networks.

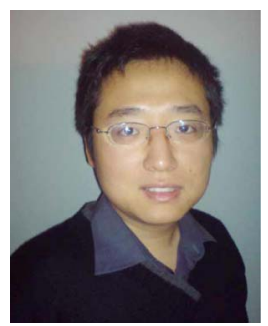

Yixuan Qin received his M.Sc. degree in computer and information networks from the University of Essex, Colchester, UK, in 2003, where he is currently working towards his Ph.D. degree. Meanwhile he is a Senior Research Officer in the Photonic Networks Lab. His research interests include high-speed digital system design, flexible networks, passive optical networks, optical burst switching, and high performance hardware accelerated computing.

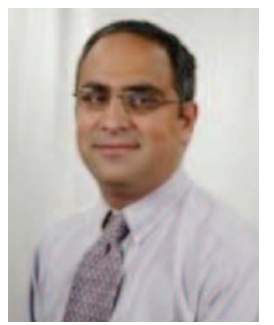

Reza Nejabati joined the University of Essex in 2002, and he is currently a member of the Photonic Network Group at the University of Essex. During the last 8 years he has worked on ultra high-speed optical networks, service oriented and applicationaware networks, network service virtualization, control and management of optical networks, and high-performance network architecture and technologies for e-science. He holds a Ph.D. in optical networks and an M.Sc. with distinction in telecommunication and information systems.

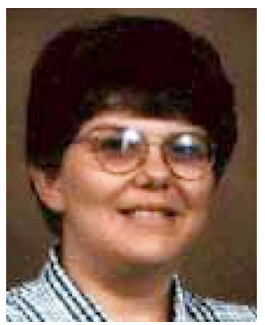

Dimitra Simeonidou is currently a Professor at the University of Essex. She has over 10 years experience in the field of optical transmission and optical networks. In 1987 and 1989 she received a B.Sc. and M.Sc. from the Physics Department of the Aristotle University of Thessalonica, Greece, and in 1994 a Ph.D. degree from the University of Essex. From 1992 to 1994 she was employed as a Senior Research Officer at the University of Essex in association with the MWTN RACE project. In 1994 she joined Alcatel Submarine Networks as a Principle Engineer and contributed to the introduction of WDM technologies in submerged photonic networks. She participated in standardization committees and was an advising member of the Alcatel Submarine Networks patent committee. Professor Simeonidou is the author over 250 papers and holds 18 patents relating to photonic technologies and networks. Her main research interests include optical wavelength and packet switched networks, network control and management, and GRID networking.

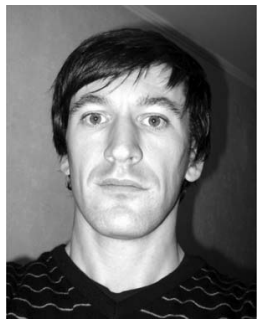

Chris Develder received the M.Sc. degree in computer science engineering and a $\mathrm{Ph} . \mathrm{D}$. in electrical engineering from Ghent University (Ghent, Belgium), in July 1999 and December 2003, respectively. From October 1999 on, he worked in the Department of Information Technology (INTEC), at the same university, as a Researcher for the Research Foundation-Flanders (FWO), in the field of network design and planning, mainly focusing on optical packet switched networks. In January 2004, he left the university to join OPNET Technologies, working on transport network design and planning. In September 2005, he re-joined INTEC at Ghent University as a Post-Doctoral Researcher, and as a Post-Doctoral Fellow of the FWO since October 2006. Since October 2007 he has held a PartTime Professor position at the same institute. He was and is involved in multiple national and European research projects (IST Lion, IST David, IST Stolas, IST Phosphorus, IST e-Photon One). His current research focuses on dimensioning, modeling, and optimizing optical grid networks and their control and management. $\mathrm{He}$ is an author or co-author of over 45 international publications. 


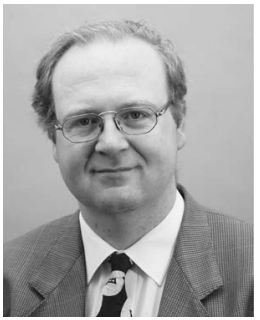

Bart Dhoedt received a degree in engineering from the Ghent University in 1990. In September 1990, he joined the Department of Information Technology of the Faculty of Applied Sciences, University of Ghent. His research, addressing the use of micro-optics to realize parallel free space optical interconnects, resulted in a Ph.D. degree in 1995. After a 2 year post-doc in opto-electronics, he became a Professor at the Faculty of Applied Sciences, Department of Information Technology. Since then, he has been responsible for several courses on algorithms, programming, and software development. His research interests are software engineering and mobile and wireless communications. He is the author or co-author of approximately 150 papers published in international journals or in the proceedings of international conferences. His current research addresses software technologies for communication net- works, peer-to-peer networks, mobile networks, and active networks.

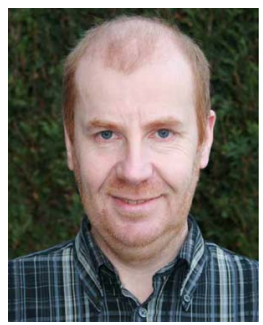

Piet Demeester received his Ph.D. degree (1988) at Ghent University, where he became a Professor in 1993. He is heading a research group on broadband communication networks and distributed software: www.ibcn.intec.ugent.be. His current research interests include multilayer networks, quality of service mobile and sensor networks, access networks, grid computing, energy efficient ICT, distributed software, network and service management, and techno-economics and applications. He is co-author of over 700 publications in international journals or conference proceedings. 